Pub. Mat. UAB

$N^{\circ} 22$ Nov. 1980

Actes VII JMHL

MODELOS MATEMATICOS EN SOLIDIFICACION: APLICACIONES EN METALURGIA.

Alfredo Bermúdez de Castro

Dpto. de Ecuaciones Funcionales

Universidad de Santiago de Compostela

Abstract.- In this paper mathematical models for heat transfer with melting or solidification are considered. These models are variants of the well-known Stefan's problem which are of considerable interest in many fields as metal casting, nuclear reactor safety, purification of materizis, etc.

INTRODUCCION .-

Los procesos de transmisión de calor con cambio de estado aparecen en numerosos campos de las ciencias aplicadas y la tecnología. A modo de ejemplo citemos la solidificación de coladas en metalurgia, la rusión del hielo y solidificación del agua, la purificación de materiales, la congelación de alimentos, el almicenamiento de la energía, etc.

Desde un punto de vista fisico los fenómenos de transmisión de calor con cambio de estado difieren de los ordinarios por el he cho de que, mientras que en estos la entalpia es una función continua, en aquellos presenta una discontinuidad de salto en la superricie de se paración de ambas fases.

Desde un punto de vista matemático, la dificultad fundamen tal estriba en que no se conoce a priori la evolución de esta superficie a lo largo del tiempo; se trata de un problema de "frontera libre".

Las diferentes formulaciones que se conocer responden al mismo objetivo: transformar las ecuaciones de partida, válidas en cada fase por separado, en una sola ecuación que tengá validez en la totalidad de la región ocupada por el cuerpo, con independencia de la posición de las fases.

Mayor dificultad se presenta cuando la temperatura de cambio de fase as variable y constituyo una incógnita adicional del proble 
ma. Asi por ejemplo, en la solidificación de ciertas aleaciones dicha temperatura depende fuertemente de la concentración de soluto, cuya evolución está regida a su vez por otro conjunto de ecuaciones en derivadas parciales.

Por si fuera poco en el momento de la solidificación tiene lugar un rechazo de soluto (ó a veces de disozvente) de la fase sólida hacia la liquida. Por consiguiente la concentración no es continua y esto es fuente de nuevas dificultades como se verá más adelante.

En este artículo se presentan algunos modelos para la simu lación (desde un punto de vista térmico) de procesos de cambio de estado. Asimismo se dan formulaciones que permiten el análisis matemático de los mismos y su resolución nuinérica.

El plan es el siguiente:

1.- El modelo de Stefan

2.- El modelo de Stefan con convección

3.- Problemas con dos materiales

4.- La soliơifcación de una aleación.

1.- El modelo de Stefan.

Con objeto de facilitar la comprensión vamos a considerar un ejemplo concreto: la solidificación del acero en colada continua (ver SAGUEZ-LARRECO ( 1 ] ). La figura 1 es una representación esquemática de una planta de colada continua. Si $\theta_{S}{ }^{y} \theta_{L}$ designan la temperatura en

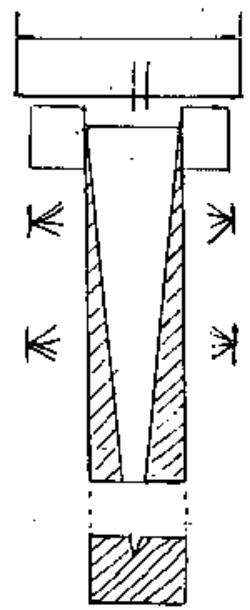

Fig. 1 las fases sólida y líquida, respectivamerte, el modelo de Stefan establece que :

$$
\begin{aligned}
& \text { En el sólido } \\
& \left.(1.1) \rho\left(\theta_{S}\right) c\left(\theta_{S}\right) \frac{\partial \theta_{S}}{\partial \tau}-\sum_{i=1}^{3} \frac{\partial}{\partial x_{i}}\left(k i \theta_{S}\right) \frac{\partial \theta_{S}}{\partial x_{i}}\right)+ \\
& +o\left(\theta_{S}\right) c\left(\theta_{S}\right) U \frac{\partial \theta_{S}}{\partial x_{3}}=0
\end{aligned}
$$

\section{En el liquido}

$$
\begin{gathered}
(1.2) \rho\left(\theta_{L}\right) \subset\left(\theta_{L}\right) \frac{\partial \theta_{L}}{\partial T}-\sum_{i=1}^{3} \frac{\partial}{\partial x_{i}}\left(k\left(\theta_{S}\right) \frac{\partial \theta_{S}}{\partial x_{i}}\right)+ \\
+o\left(\theta_{L}\right) c\left(\theta_{L}\right) U \frac{\partial \theta_{L}}{\partial x_{3}}=0
\end{gathered}
$$

\section{En la superficie de separación}

(1.3) $\theta_{S}=\theta_{L}=\bar{\theta} \quad$ (temperatura de solidificación).

(1.4) $\quad k\left(\theta_{S}\right) \overrightarrow{\operatorname{grad}} \theta_{S} \cdot \vec{N}-k\left(\theta_{L}\right) \operatorname{grad} \theta_{L} \cdot \vec{N}=p(\vec{\theta}) L \vec{V} \cdot \vec{N}$ 
Siencio U la velocidad de deccerso de la colada, L el caj..r latente de fusión, $\vec{V}$ la velocidao de avarice de la interfaste y $\vec{N}$ un vector unitario normal a ésta.

A las ecuaciones $(1.1)-(1.4)$ habría que añadir las conjicio nes de contorno y la condición tinicial.

Si suponemos que el proceso se encuentra en estado estacionario los términos con derivadas temporales desaparecen. Adenás la trans misión de calor por conducción en la dirección de avance $x_{3}$ puede despré ciarse frente a la que tiene lugar por convección de manera que las ecua ciones $(1.1)(1.2)$ se reducen a

En el sólido

$$
\rho\left(\theta_{S}\right) \operatorname{loc}\left(\theta_{S}\right) \cup \frac{\partial \theta_{S}}{\partial x_{3}}-\sum_{i=1}^{2} \frac{\partial}{\partial x_{i}}\left(k\left(\theta_{S}\right) \frac{\partial \theta_{S}}{\partial x_{i}}\right)=0
$$

En el líquido

$$
\rho\left(\theta_{L}\right) c\left(\theta_{L}\right) \cup \frac{\partial \theta_{L}}{\partial x_{3}}-\sum_{i=1}^{2} \frac{\partial}{\partial x_{i}}\left(k\left(\theta_{L}\right) \frac{\partial \theta_{L}}{\partial x_{i}}\right)=0
$$

Nótese que (1.5) (1.6) son ecuaciones de tipo parabólico, por consiguién te tomando como condición inicial ien $x_{3}=0$ ) la temperatura de vertido del acero, podemos integrarlas paso a paso y seguir la evolución de la temperatura de una sección en su descenso.

La ecuación $(1.4)$ deberá sustituirse por

$$
k\left(\theta_{S}\right) \sum_{i=1}^{2} \frac{\partial \theta_{S}}{\partial x_{i}}: n_{i}-k\left(\theta_{L}\right) \sum_{i=1}^{2} \frac{\partial \theta_{L}}{\partial x_{i}} \cdot n_{i}=\rho(\bar{\theta}) c(\bar{\theta}) L U \vec{v} \cdot \vec{n}
$$

donde $\vec{v}$ es la velocidad de avance de la interfase en el plano $x_{1} x_{2}$ respecto $a x_{3}$ y $\vec{n}=\left(n_{1}, n_{2}\right)$ es ur vector unitario en la dirección $\left(N_{1}^{1}, N_{2}\right)$.

Existen fundamentalmente tres formulaciones oel problema de Stefan (1.5)-(1.7) que comentamos brevemente a continuación. Por las razones apuntadas más arriba escribiremos $t$ en lugar de $x_{3} y \quad x=\left(x_{1}, x_{2}\right)$.

i) Formulación en temperatura.- Sea $\Omega$ el abierto de $R^{n}$ ocu pado por el material y $Q=\Omega \times] 0, T[$. Para $t \in] O, T\left[\right.$ se denotan $\Omega_{S}(t), \Omega_{L}(t)$ los subconjuntos de $\Omega$ ocupadọs por las fases sólida y líquida respectiva mente $y$ se define en $Q$ una función $\theta$ del siguiente modo:

$$
\theta(x, t)=\left\{\begin{array}{lll}
\theta_{S}(x, t) & \text { si } & x \in \Omega_{S}(t) \\
\theta_{L}(x, t) & \text { si } & x \in \Omega_{L}(t)
\end{array}\right.
$$

Entonces, bajo ciertas hipótesis de regularidad y mediante formulas de Green no es dificil probar (ver LIONS (2)) que o verifica 
Siendo $H$ el operador maximal monótono en $R$.

$(1.10)$

$$
H(\theta)= \begin{cases}\int_{0}^{\theta} \rho(y)(y) d y & \text { si } \theta<\bar{\theta} \\ {\left[\int_{0}^{\theta} \rho(y) c(y) d y, \int_{0}^{\theta} \rho(y) c(y) d y+p(\bar{\theta}) L\right]} & \text { si } \theta=\bar{\theta} \\ o(\bar{\theta}) L+\int_{0}^{\theta} \rho(y) c(y) d y & \text { si } \theta>\bar{\theta}\end{cases}
$$

$H(\theta(x, t))$ representa la entalpia local del purto $x$ en el instante $t$, de modo que (1.9) expresa algo evidente desde el punto de vista físico: la variación de entalpía local por unidad de tiempo es igual al calor que se transmite por conducción.

Es bien conocido que cuatido el húmero de variables espaciałes es superior a uno, la existencia de solución clásica del problema de Stefan es una cuestión abierta. Por este motivo numerosos autores han considerado una formulación débil obtenida a partir de (1.9), para la que prueban resultados de existencia y unicidad (ver OLEINIK [3], KAMENOMOTSKAYA [4], LADYZENSKAiA-SOLONNIKOV-URALTCEVA [5], FRIEDMANN [6]).

Un problema más difícil es el da la regularidad de la solu ción débil y de la frontera libre. Aqui de nuevo los resultados obtenidos dependen. de la dimensión espacial. Si ésta es uno y no hay fuentes

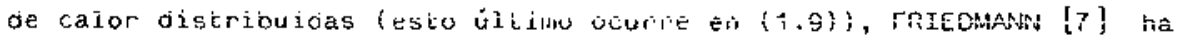
probado la continuidad de la solución débil, y el mismo autor en [8] la analiticidad de la frantera libre.

En dimensión superior a uno los resultados que se conocen en este sentido, lo son para el llamado problema con una fase, que consiste en considerar que la temperatura en una de las fases es constante e igual a la temperatura de cambio de estado. En este caso FRIEDMANNKINDERLEHRER [9] han denostrado un resultado de regularidad para la frontera libre y más recientemente CAFFARELLI-FRIEDMANN [10] la continuidad de la solución débil.

En cuanto a los métodos numéricos existen varios que parten de la formulación en temperatura. Dejando a un lado el caso unidimensional para el cual existe una abundante bibliografía, merece destacarse en primer lugar el artículo de kAMENOMOSTKAYA [4] donde se prueba la convergencia del esquema explícito. Por su parte MEYeR [11] conside ra un problema regularizado lobtenido al aproximar $H$ por una familia de funciones continuas) para el que orueba la convergencia del esquema impícito.

También SAGUEZ-LARRECQ [1] discretizan en tiempo el proble ma mediante un esquema implicito, aunque sin regularización previa de la función $H$. En cada paso de tiempo resuelven la ecuación obtenida con un algoritmo de multiplicadores de BERMUDEZ-MORENo \{12\}. 


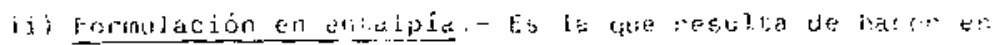

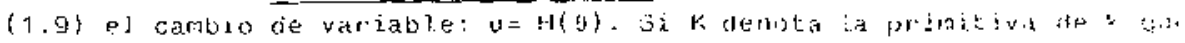
jasa por is o! igst se tiene:

$$
(1 . \vec{i}) \quad U \frac{\partial u}{\partial i}-\Delta\left(k \mathrm{H}^{-;}\right) u=0
$$

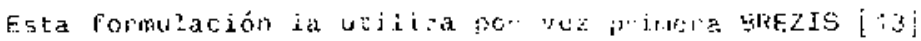

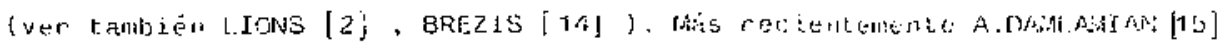

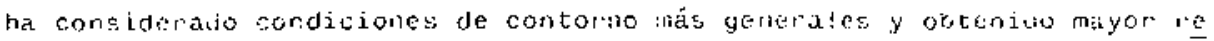
gularijal. Sus resultados son aplicaciones de la teoria de semigrupos.

La resolución numérica de $(1,11)$ se abonda en los avticulos de CIAVALOINI [16], DEGUEIL [17;BREZZiS-ROGERS-RERGER [18] et:.

iii) Formulación con inecuaciones variacionales.- Ha sido in troducida por DUVAUT [19] para el problema con una fase y por DIJVAT [20] y FREOMOND [21], separadamente, para el problema con dos fases. En ambos casos se define una nueva incógnita "grosso modo" la integrel en tiempo de la lemperatura) la cual es solucjón ce una inecuación variacional de evolución de primera o segunda especie según que el aúmero de fases sea uno o dos.

Resultados numéricos sobre esta formulación pueden verse e $\pi$ AGUIRRE -FREMOND [22], SAGUEZ [23].

2.- El modelo de Stefar con convección. (ver BERMUOEZ-SAGUEZ i 24 )).

El modelo $(1.1)-(1.4)$ supone que la transmisión de calor en las direcciones $x_{1} \dot{0} x_{2}$ se realiza solamente por conducción. Ahora bien existen casos (como el de la colada continua a nivel de la lingotera), en los que tienen lugar movimientos en la fase líquida que obli gan a considerar la transmisión por convccción, incroctuciendo en (1.2) wr término suplementario. Procediendo de este modo, en lugar de $(1.9)$ se obtiene la ecuación:

$$
U \frac{\partial H(\theta)}{\partial t}-\operatorname{div}(k(\theta) \overrightarrow{\operatorname{grad}} \theta)+c(\theta) c(\theta) \vec{b} \cdot \operatorname{grad}(\theta-\bar{\theta})^{+}=0
$$

En BERMUDEZ-SAGUEZ [24]' se prueban resultados de existencia y unidad de solución para una ecuación de evolución no lineal que incluye a $(2.1)$ como caso particular.

3.- Probiemas con dos materiales.

Cuando una masa de metal fundido se enfría en un molde, la parte que solidifica en último lugar sufre una contracción debido al aumento de densidad, originándose una cavidad liamada "rechupe" iver p. ej. RUDOLE [25]). Es cia gran interés predecir la situación del rechupe con objeto de evitarlo o al menos desplazarlo a otro lugar, 10 cual ouede realizarse nodificando el poder enfriador de ciertas partes del moloé ó disporiendo "mazarotas" que alimenten de metal liquido las partes que solidifican en ultimo lugar. 
Veamos como el modelo de Stefan puede adaptarse para simular este proceso. Primeramente nótese que ahora nos interesa el fenómeno transitorio, asi que no se podrán hacer las simplificaciones del \$1, y por tanto, salvo casos especiales con ciertas simetrias (p. ej. en jiezas ailindricas), el dominio $\Omega$ será tridimensional.

Sean $\Omega_{1}$ y $\Omega_{2}$ las regiones ocupadas por el metal fundido $y$ el molde, respectivamente, y $\Omega=\Omega_{1} \cup \Omega_{2}$ (ver fig. 2 ).

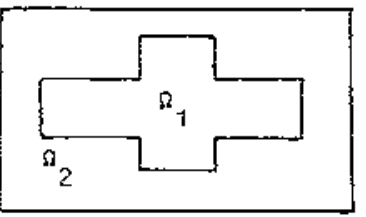

La diferencia con los problemas en los que interviene un solo material es que ahora la entalpia depende no sólo de la temperatura sinu también de $x$. Concreta mente:

Fig. c

$$
\int_{0}^{\theta} \nu_{1}(y) c_{1}(y) d y
$$

$(3,1)$ si $x \in \Omega_{1}: H(x, \theta)=\left\{\int_{0}^{\theta} \rho_{1}(y) c_{1}(y) d y, \int_{0}^{-} \rho_{1}(y) c_{1}(y) d y+\rho(\bar{\theta})\llcorner\} \theta=\bar{\theta}\right.$

$$
\rho(\bar{\theta}):=\left\{\begin{array}{l}
\theta \\
0
\end{array} \rho_{1}(y) c_{1}(y) d y\right.
$$

$\theta>\bar{\theta}$

$$
\text { si } x \in \Omega_{2} \quad H(x, \theta)=\int_{0}^{\theta} \rho_{2}(y) c_{2}(y) d y
$$

Utro problema con dos materiales, gue surge en el estudio de reactores nucleares (ver EL GENK-CRONENBERG [26]), es el siguiente:

Se trata de transportar un líquido a alta temperatura, a través de una tubería construida con otro material cuyo punto de fusión es más bajo que el de solidificación del líquido. Se comprende que la región interior de la tubería va a fundirse, al tiempo que una capa de liquido en contacto con ella se solidifica. Para evitar la fusión total de la tubería, esta se refrigera en su exterior con un liquido frío.

Como èn el ejemplo anterior, la diferencia con el problema clásico de Stefan está en la función entalpia que ahora viene dada por:

$$
\int_{0}^{\theta} o_{i}(y) c_{i}(y) d y
$$

(3.3) $x \in \Omega_{i} H(x, \theta)=\left[\int_{0}^{\bar{\theta}_{i}} \rho_{i}(y) c_{i}(y) d y, \int_{0}^{\bar{\theta}_{i}} \rho_{i}(y) c_{i}(y) d y+\rho_{i}\left(\bar{\theta}_{i} H_{i}\right] \theta=\bar{\theta}_{i}\right.$

$$
\int_{0}^{\theta} \rho_{i}(y) c_{i}(y) d y+o_{i}\left(\bar{\theta}_{i}\right) L_{i}
$$

donde $\bar{\theta}_{i}, L_{i}, s_{i}(i=1,2)$ designan, respectivamente, la temperatura de fusión, el calor latente y las regiones acupadas por el líquido que se transporta $(i=1)$ y la tuberia $(i=2)$. 


\section{4. - Solidificación de una aleación.}

Como se ha dicho en la introducción, en este caso la tempe ratura de solidificación depende de la concentración de soluto fver CHALMERS $(27])$. Denotando por $C_{s}$ (resp. $C_{L}$ ) dicha concentración en la fa se sólida (resp. liquida), se tendrá:

En el sólido

$$
\frac{a C_{S}}{a t}-D_{S} \Delta C_{S}=0 \quad\left(D_{S}: \text { coef. de difusión }\right)
$$

En el liquido

$$
\frac{\partial C_{L}}{\partial t}-D_{L} \Delta C_{L}=0 \quad\left(D_{L}: \text { coef, de difusión }\right)
$$

Además la concentración no es una función continua en la interfase: la composición del sólido que se forma difiere de la del liquido en equili brio con él, como muestra el siguiente diagrama. Su interpretación es

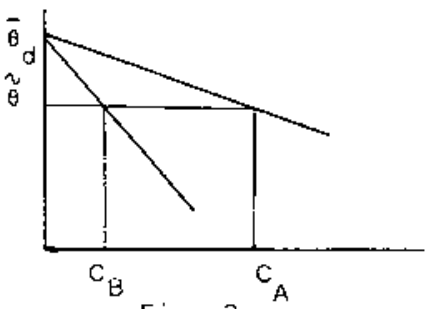

Fig. 3 sencilla. Sea $C_{A}$ la concentración de un punto de la aleación en estado líquido, que se enfria progresivamente. Cuando se alcance la temperatura $\ddot{b}$, dicho punto se solidificará $y$, en ese momento, rechazará soluto hacia la zona liquida hasta que su concentración sea $C_{B}$. Si $\frac{1}{I_{S}}$ y $\frac{1}{\sigma_{L}}$ son las pendientes de las rectas de la figura, la ley de conserva ción de la masa impone la siguiente ecuación en la interfase:

(4.3) $D_{S} \overrightarrow{\operatorname{grad}} C_{i j} \cdot \vec{N}-D_{L} \overrightarrow{\operatorname{grad}} C_{L} \cdot \vec{N}=\left(\sigma_{S}-\sigma_{L}\right)\left(g-\bar{\theta}_{d}\right) \vec{V} \cdot \vec{N}$

donde $\bar{\theta}_{\mathrm{d}}$ es la temperatura de solidificsción del disolvente y g la temperatura a la que solidificará cada punto de $\Omega$.

Para resolver (4.1)-(4.3), FIX [28] sugiere la introducción de la nueva función incógnita:

$$
w=\left\{\begin{array}{l}
w_{S}=\frac{C_{S}}{\sigma_{S}}+\bar{\theta}_{d} \text { en la fase sólida } \\
w_{L}=\frac{C_{L}}{\sigma_{L}}+\bar{\theta}_{d} \text { en la fase liquida }
\end{array}\right.
$$

que tiene un significado físico preciso " $W(x, t)$ es la temperatura a la que cambiaria de estado el punto $x$ a la vista de la concentración que tiene en el tiempo t". Por consiguiente, la interfase en el tiempo t se rá: 
y la función y vendráa dada por:

$$
g(x)=W(x, t) \quad \text { si } x \in R(t)
$$

A partir de (4.1)-(4.3) no as efifícil probar que wes solu ción de la ecuación

$$
\frac{a G_{\theta}(w)}{\partial t}-\operatorname{div}\left(M_{\theta}(w) \text { grad } w\right)=0
$$

donde ia $y$ is vienen dadas por:

(4.8)

$$
G_{\theta}(w)= \begin{cases}-\sigma_{L}\left(\forall i-\bar{\theta}_{d}\right) & \text { si } w<\theta \\ {\left[-\sigma_{L}\left(\theta-\bar{\theta}_{d}\right),-\sigma_{S}\left(e-\bar{\theta}_{d}\right)\right]} & \text { si } w=\theta \\ -\sigma_{S}\left(W-\vec{\theta}_{d}\right) & \text { si } w>\theta\end{cases}
$$

$$
M_{\theta}(W)= \begin{cases}-\sigma_{i} D_{L} & \text { si } w<\theta \\ -\sigma_{S} D_{S} & \text { si } w>\theta\end{cases}
$$

Por otra parte la ecuación que rige la evolución de la tem peratura continúa siendo $(1.9) \quad 0(2.1)$ (con $U=1$ ) pero la entalpia local es ahora función de it:

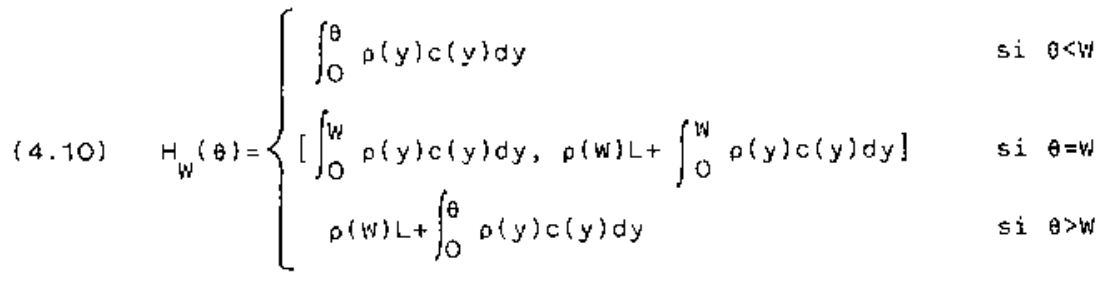

La existencia de una solución para las ecuaciones (4.8) y (1.9) con $H$ definida por (4.10), asi como el desarrollo de métodos numéricos para su resolución con justificación matemática, parecen cuestiones abiertas. No obstante, con respecto a este ultimo tema, las ioeas contenidas en el articulo de FIX [28] junto con el algoritmo de BERMUDEZ-MORENO [12] para resolver problemas multivocos conducen a algoritmos convergentes como parecen indicar algunas experiencias realiza das con ordenador (BERMUDEZ-SAGUEZ [29]). 


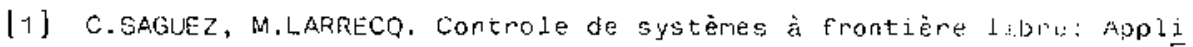
cation à la coulée continue dacier. $4^{\text {erre }}$ coi. lili. sur les Méthodes de Calcul Sc. et Technique. Vursailles (i979).

[2] J.L.LIONS. Quelques méthodes de resolution de problènes aux limites nonlinéaires. Dunod. Paris (1969).

[3] 0.OLEINIK. A method of solution of the general stefan problem. Soviet Math. Dokl. $1(1960)$ p. 1350-1354.

[4] S.L.KAMENOMOSTKAYA. On Stefan's problem. Math. Sb. 53 (1965) p. 485-514.

[5] O.LADIZENSKAYA, V.SOLONNIKOV, N.URALTCEVA. Linear and quasilinear equations of parabolic type. Transl. Math. Monographs, vol. 23, A.M.S. Providence (1968).

[6] A.FFIEOMANN. The Stefan $p r o b l e m$ in several space variables. Trans. Amer. Math. Soc. $133(1968)$, p. 51-87.

[7] A.FRIEDMANN. One dimensional Stefan problems with nonmonotone free boundary. Trans. Amer. Math. Soc. 133 (1968),p. 89-114.

[8] A.FRIEDMANN. Analiticiy of the free boundary for the Siefan problem. Arch. Rat. Mech, and Analysis. 61 (1976), p. 9i-125.

[9] A.FRIFOMANN, D.KINDERLEHRER. A one phase Stet́an froblem. Ind. Univ. Math. J. 24 (1975), p. 1005-1035.

[10] L.CAFFARELLI, A.FRIEDMANN. Continuity of the temperature in the Ste fan problem. Ind.Univ.Math.J. 28 (1979), p. 53-70.

[11] G.H.MEYER. Multidimensional Stefan problems. SIAM J. Num. Anal. 10 $(1973), p .522-538$.

[12] A. BERMUDEZ, C.MORENO. Duality methods for solving variational inequalities. Comp. and Math. with. Appl. (1980).

[13] H.BREZIS. On some degenerate parabolic equations. Proc.symp. on non Iinear functional analysis. Chicago (1968) de Proc. Symp. Pure Math. AMS (1970).

[14] H.BREZIS. Monotonicity methods in Hilbert spaces and some applications to nonlinear partial differential equations. En Contributions to nonlinear functional analysis. Ed. E.H.ZARANTONELLO, Academic Press (1971).

[15] A.DAMLAMIAN. Some results on the multi-phase Stefan problem. Commun. Partial Differ. Eq. 2 (1977), p. 1017-1044.

[16] J.CIAvaldinI. Analyse numérique d'un problème de stefan à deux phases par une methode d'éléments finis. SIAM J. Num. Anal, 12 (1977). 
[17] A.DEGUEIL. Fesolktion par Lung nethode d'elements finis d'un problème ce sefefan en remies de temperature et teneur en malerian non gélé. Tesis $3^{e r}$ eiclo. Univ. Bordeaux I (1977).

[18] H.BREZIS, J.C.W.ROEERS, A.E. BERRER. A numerical method for solving the probiem $u_{t}-\Delta f(u)=0$.

[19] G.DUvaut. Resolution d'un problème de Stefan. C.R. Acad. Sci. Paris, $276(1973)$, p. 1461-1463.

[20] G.Duvaut. Problèmes à frontière libre en théorie des milieux continus, Rapport du LABOR'IA ne 185 (1976).

[21] M.FREMOND. Frost propagation in porous media. Int. Conf . on Comp. Methods in nonlinear Mechanics. Austin (1974).

[22] J.AGUIRRE, M,FREMONO. Froscpropagation in wet porous media en Applications of Methods of Functional Analysis in Mechanics. Lecture Notes in Mathematics ne 503. Springer.

[23] C.SAGUEZ. Estudio de probiemas con frontera libre: Simulación y control ćptimo. Publicaciones del Dpto. de Ecuaciones Funcionales. Universidad de Santiago (1980).

[24] A.BERMUDEZ, C.SAGJEZ. Une équation non-linéaire intervenant en solidification. (De próxima apariciónj.

[25] R.W.RUOCLE. The solidificalion of castings. The Institute of Metals. tondon (1957).

[20] M.S. EL-GENK, A.W.CRONENBERG. Stefar-like problems in finite geometry. AICHE Symp. Series. Heat Transfer-San Diego (1979).

[27] B.CHALMERS. Principles of solicificatior. John Wiley New York $(1964)$.

[26] G.J.FIX. Numerical methods for alloy solidification problems. En Moving boundary problems. Ed. D.G.WILSON, A.D.SALOMON, P.T.BOGGS. Academic Press, New York (1978).

[29] A.Bermudez, C.Saguez. Trabajo sin publicar. 